\title{
Evaluation of Risk Factors for Amputation and Mortality in Patients of Necrotizing Fasciitis
}

\author{
Maulik Sharma ${ }^{1}$, Rajkamal Kanojiya², Shefali Goyal ${ }^{3}$
}

\begin{abstract}
Background: Necrotizing fasciitis (NF) is a serious infection of the skin and soft tissues including subcutaneous tissue and fascia, which rapidly progresses along the deep fascia and becomes a medical and surgical urgency. Delayed treatment is associated with loss of limb and infection and is the most common cause of mortality. The purpose of our study is to identify the risk factors which may be used to predict amputation and mortality in patients with NF.

Materials and methods: All adult patients presented in General Surgery OPD/emergency and plastic surgery OPD of MGUMST with NF were assessed, admitted, and treated with broad-spectrum antibiotics from January 2019 to June 2020. All routine blood parameters were evaluated and were provided proper emergency surgical treatment. The surgical interventions included incision and drainage, debridement, fasciotomy, and amputation. The clinical data and demographic characteristics within the first day of admission and after 48-72 hours were documented. Important data associated with the investigations and treatment of NF, i.e., wound appearance, site of infection, organisms involved, and laboratory data within the first day of admission and after 48-72 hours, surgical intervention and outcome, were documented. Patients were divided into two groups: survival and mortality which were further grouped into two groups: those with amputation and without amputation. Results: Among 100 patients with diagnosis of NF, 20\% died and $10 \%$ underwent amputation. From multivariable analysis, the predictors of mortality among patients of NF were patients of age $>60$ years [risk ratio $(\mathrm{RR})=58.57,95 \% \mathrm{Cl}=3.64-941.85, p=0.004$ ], having diabetes mellitus $(\mathrm{DM})(\mathrm{RR}=28.70,95 \% \mathrm{Cl}=1.78-461.51, p=0.017)$, gangrene $(\mathrm{RR}=3.86,95 \% \mathrm{Cl}=1.91-7.75, p=0.0002)$, severe sepsis $(\mathrm{RR}=158.14,95 \% \mathrm{Cl}=$ 9.96-2508.89, $p=0.0003)$, involvement of multiple sites $(R R=9.06,95 \% \mathrm{Cl}=4.25-19.31, p=0.0001)$, total leukocyte count $(\mathrm{RR}=158.14,95 \%$ $\mathrm{Cl}=9.96-2508.89, p=0.0003)$, and serum creatinine $(\mathrm{RR}=126.28,95 \% \mathrm{Cl}=7.92-2013.12, p=0.0006)$ on admission and within $48-72 \mathrm{hours}$ of admission and the independent predictors of amputation among patients of $\mathrm{NF}$ included having gangrene $(\mathrm{RR}=173.73,95 \% \mathrm{Cl}=10.91-2764.80$, $p=0.0003)$, severe sepsis ( $R R=6.00,95 \% \mathrm{Cl}=1.86-19.26, p=0.002)$, total leukocyte count $(\mathrm{RR}=6.00,95 \% \mathrm{Cl}=1.86-19.26, p=0.002)$, and serum creatinine $(\mathrm{RR}=64.68,95 \% \mathrm{Cl}=3.92-1064.68, p=0.003)$ on admission and within $48-72$ hours of admission.

Conclusion: In our study, we concluded that gangrene, severe sepsis, total leukocyte count $>25,000$, and serum creatinine values $>1.6 \mathrm{mg} / \mathrm{dL}$ on admission as well as within 48-72 hours, as independent predictors for both amputation and mortality and some other factors like age $>60$ years, the involvement of multiple sites, DM mainly predicting the mortality rate independently.

Keywords: Amputation, Debridement, Fasciotomy, Gangrene, Necrotizing fasciitis.

Journal of Mahatma Gandhi University of Medical Sciences \& Technology (2020): 10.5005/jp-journals-10057-0130
\end{abstract}

\section{INTRODUCTION}

Necrotizing fasciitis (NF) is a rare, sometimes lethal, bacterial infection characterized by widespread skin, superficial fascia, and subcutaneous tissue necrosis. ${ }^{1,2}$ The incidence has been reported between 0.4 and 0.53 cases per 100,000 population. ${ }^{3-5}$ Now, an increasing incidence of NF has been reported, which can be attributed by a better reporting system and an increment in strains which are associated with NF because of unrational use of antibiotics. Patients presenting with preexisting factors, trauma, many young individuals may also be affected by this disease. Approximately half of the cases of NF (caused by streptococcal) are found in young and previously healthy patients. ${ }^{6}$ The overlying skin appears normal but it is the necrotic process that works underneath. Later on, the patient becomes extremely toxic and presents with painful, red as well as necrotic skin as blood supply to the skin is gradually hampered. Pathophysiologically, it is caused by septic thrombosis of the vessels that are between skin and the deeper layers.

Necrotizing fasciitis results from either trauma to the skin or from hematogenous spread. ${ }^{1}$ Any kind of trauma whether major or minor which breaches skin integrity including needle punctures,
${ }^{1-3}$ Department of General Surgery, Mahatma Gandhi University of Medical Sciences and Technology, Jaipur, Rajasthan, India

Corresponding Author: Maulik Sharma, Department of General Surgery, Mahatma Gandhi University of Medical Sciences and Technology, Jaipur, Rajasthan, India, e-mail: chalanasrishty7@gmail. com

How to cite this article: Sharma M, Kanojiya R, Goyal S. Evaluation of Risk Factors for Amputation and Mortality in Patients of Necrotizing Fasciitis. J Mahatma Gandhi Univ Med Sci Tech 2020;5(2):38-46.

Source of support: Nil

Conflict of interest: None

bites from insects, burns, lacerations, surgical wound, blunt trauma, etc., may develop this aggressively spreading, insidiously advancing soft-tissue condition., ${ }^{1,2}$

Necrotizing fasciitis occurs in any part of the body but primarily it involves the superficial layers of the extremities, abdomen, or perineum. ${ }^{7}$

(0) The Author(s). 2020 Open Access This article is distributed under the terms of the Creative Commons Attribution 4.0 International License (https:// creativecommons.org/licenses/by-nc/4.0/), which permits unrestricted use, distribution, and non-commercial reproduction in any medium, provided you give appropriate credit to the original author(s) and the source, provide a link to the Creative Commons license, and indicate if changes were made. The Creative Commons Public Domain Dedication waiver (http://creativecommons.org/publicdomain/zero/1.0/) applies to the data made available in this article, unless otherwise stated. 
The various presentations of microbial involvement in these tissues may include simple contamination and self-limited bacterial contamination to an unrelapsing, unpredictable clinical course and projecting to septicemia, multiple organ failure, and death. ${ }^{8}$

The purpose of our study is to identify the risk factors which may be used to predict amputation and mortality in patients with NF.

\section{Aims and OBjectives}

To evaluate the risk factors for amputation and mortality in patients with NF.

\section{Materials and Methods}

- Type of study: prospective cohort study.

- Period of study: January 2019 to June 2020.

- Place of study: General Surgery OPD, Mahatma Gandhi Medical College and Hospital, Jaipur.

- Ethical clearance: Institute Ethics Committee approval was obtained before the start of the study.

- Written and informed consent was obtained from all participants before enrolment into the study.

\section{Sample Size}

The study included 100 adult patients presenting with NF in OPD/ emergency of general surgery department/plastic surgery OPD/ emergency of Mahatma Gandhi Medical College and Hospital, Jaipur of all surgical units.

\section{Plan of Study}

Patients presenting to surgical OPD/plastic surgery OPD/emergency with features of NF were evaluated.

\section{Inclusion Criteria}

All the adult patients presenting with features of NF will be included in the study.

\section{Exclusion Criteria}

- Unwilling patients.

- Patients with age $<18$ years.

\section{Methods}

All adult patients presented in General Surgery OPD/emergency and plastic surgery OPD of MGUMST with NF were assessed, admitted, and treated with broad-spectrum antibiotics. All routine blood parameters including complete blood counts, renal and hepatic function tests, coagulation profile, and random blood sugar were evaluated and were provided proper emergency surgical treatment. The surgical interventions included incision and drainage, debridement, fasciotomy, and amputation. The clinical data and demographic characteristics including sex, age, body mass index, education, occupation, underlying disease, vital signs within the first day of admission and after 48-72 hours were documented. Important data associated with the investigations and treatment of NF, i.e., wound appearance, site of infection, organisms involved, and laboratory data within the first day of admission and after 48-72 hours, surgical intervention and outcome, were documented. Patients were divided into two groups: survival and mortality which were further grouped into two groups: those with amputation and without amputation.

\section{Statistical Analysis}

The collected data were analyzed with IBM. SPSS statistics software 23.0 Version. For the description of the data, descriptive statistics frequency analysis and percentage analysis were used for categorical variables, and the mean and SD were used for the continuous variables. To find the significant difference between the bivariate samples in independent groups the unpaired sample $t$-test was used. To find the significance in categorical data, Chi-square test was used similarly if the expected cell frequency is $<5$ in $2 \times 2$ tables then the Fisher's exact was used.

In the above statistical tools, the probability value of 0.05 was considered significant.

\section{Observations and Results}

A total of 100 patients with NF were enrolled in this study and various factors including predisposing factors, age, wound appearance, laboratory investigations, and wound appearance were taken into account that possibly affect the outcome of NF and its morbidity and mortality.

Among 100 patients in our study, 59 patients were above 60 years of age and 41 patients were below 60 years of age and most of the patients were males (65) as compared to females (35). Regarding the comorbid conditions causing NF, the commonest being diabetes mellitus (DM) in 59\% cases, followed by gout, coronary heart disease, renal disease, and cirrhosis. Regarding the clinical presentation, the commonest symptom being swelling and is present in about $95 \%$ of cases. Next to swelling is redness and severe pain in 90 and $75 \%$, respectively. Among the 100 patients, we had studied, the most common part to be affected was the lower limb (35\%), next to it was upper limbs (22\%), and involvement of trunk in $15 \%$ of patients. By blood investigations from our study, we noticed that $36 \%$ of patients had total leukocyte count $>25,000$ on admission which decreases to $20 \%$ after $48-72$ hours of admission and $39 \%$ had elevated serum creatinine on admission which reduces to $24 \%$ after $48-72$ hours.

In about $40 \%$ of patients, we performed incision and drainage and in $30 \%$ of patients, we performed wound debridement. In about $20 \%$, we had done fasciotomy and amputation in $10 \%$ of patients. Seventeen percent of patients developed severe sepsis. Most of the patients discharged after 20 days of hospital stay. And their mortality in our study was $20 \%$. Following are the tables showing a comparison of factors between mortality and survival and another table showing a comparison of factors between amputation and non-amputation (Tables 1 to 4).

\section{Comparison of Factors between Mortality and Survival Group}

Patient demographic and clinical characteristics including age ( $>60$ years), underlying morbidity like DM, wound appearance such as redness, blebs, gangrene, severe pain, involvement of multiple sites, WBC count $(>25,000)$, serum creatinine level $(>1.6)$ on admission and within 48-72 hours after admission, severe sepsis and length of hospital stay showed statistically significant differences ( $p$ value $<0.05$ ) between mortality and survival groups. Other factors did not differ significantly between the two groups. 
Table 1: Comparison of factors between mortality and survival group

\begin{tabular}{|c|c|c|c|c|c|c|c|}
\hline & & & \multicolumn{2}{|c|}{ Mortality } & \multirow[b]{2}{*}{ Total } & \multirow[b]{2}{*}{$x^{2}$-value } & \multirow[b]{2}{*}{$p$ value } \\
\hline & & & Alive & Dead & & & \\
\hline \multicolumn{8}{|c|}{ Comparison of age with mortality } \\
\hline \multirow[t]{4}{*}{ Age } & $<60$ & Count & 59 & 0 & 59 & 35.976 & $0.0005^{* *}$ \\
\hline & & $\%$ & $73.8 \%$ & $0.0 \%$ & $59.0 \%$ & & \\
\hline & $>60$ & Count & 21 & 20 & 41 & & \\
\hline & & $\%$ & $26.3 \%$ & $100.0 \%$ & $41.0 \%$ & & \\
\hline \multicolumn{8}{|c|}{ Comparison of gender with mortality } \\
\hline \multirow[t]{4}{*}{ Gender } & Female & Count & 26 & 9 & 35 & 1.099 & $0.295^{\#}$ \\
\hline & & $\%$ & $32.5 \%$ & $45.0 \%$ & $35.0 \%$ & & \\
\hline & Male & Count & 54 & 11 & 65 & & \\
\hline & & $\%$ & $67.5 \%$ & $55.0 \%$ & $65.0 \%$ & & \\
\hline \multicolumn{8}{|c|}{ Comparison of underlying morbidities with mortality } \\
\hline \multirow[t]{2}{*}{ Diabetes mellitus } & & Count & 39 & 20 & 59 & 17.373 & $0.0005^{* *}$ \\
\hline & & $\%$ & $48.8 \%$ & $100.0 \%$ & $59.0 \%$ & & \\
\hline \multirow[t]{2}{*}{ Coronary heart disease } & & Count & 9 & 1 & 10 & 0.694 & $0.405^{\#}$ \\
\hline & & $\%$ & $11.3 \%$ & $5.0 \%$ & $10.0 \%$ & & \\
\hline \multirow[t]{2}{*}{ Renal disease } & & Count & 6 & 0 & 6 & 1.596 & $0.597^{\#}$ \\
\hline & & $\%$ & $7.5 \%$ & $0.0 \%$ & $6.0 \%$ & & \\
\hline \multirow[t]{2}{*}{ Cirrhosis } & & Count & 5 & 0 & 5 & 1.316 & $0.580^{\#}$ \\
\hline & & $\%$ & $6.3 \%$ & $0.0 \%$ & $5.0 \%$ & & \\
\hline \multirow[t]{2}{*}{ Gout } & & Count & 14 & 1 & 15 & 1.961 & $0.292^{\#}$ \\
\hline & & $\%$ & $17.5 \%$ & $5.0 \%$ & $15.0 \%$ & & \\
\hline \multirow{3}{*}{ Swelling } & & Compa & vound ap & with mo & & & \\
\hline & & Count & 75 & 20 & 95 & 1.316 & $0.580^{\#}$ \\
\hline & & $\%$ & $93.8 \%$ & $100.0 \%$ & $95.0 \%$ & & \\
\hline \multirow[t]{2}{*}{ Redness } & & Count & 76 & 14 & 90 & 11.111 & $0.004^{* *}$ \\
\hline & & $\%$ & $95.0 \%$ & $70.0 \%$ & $90.0 \%$ & & \\
\hline \multirow[t]{2}{*}{ Bleb } & & Count & 40 & 0 & 40 & 16.667 & $0.0005^{* *}$ \\
\hline & & $\%$ & $50.0 \%$ & $0.0 \%$ & $40.0 \%$ & & \\
\hline \multirow[t]{2}{*}{ Skin necrosis } & & Count & 23 & 7 & 30 & 0.298 & $0.585^{\#}$ \\
\hline & & $\%$ & $28.8 \%$ & $35.0 \%$ & $30.0 \%$ & & \\
\hline \multirow[t]{2}{*}{ Gangrene } & & Count & 4 & 6 & 10 & 11.111 & $0.004^{* *}$ \\
\hline & & $\%$ & $5.0 \%$ & $30.0 \%$ & $10.0 \%$ & & \\
\hline \multirow[t]{2}{*}{ Severe pain } & & Count & 55 & 20 & 75 & 8.333 & $0.003^{* *}$ \\
\hline & & $\%$ & $68.8 \%$ & $100.0 \%$ & $75.0 \%$ & & \\
\hline & & Comparis & een the si & Ind with n & & & \\
\hline Head and neck & Present & Count & 10 & 0 & 10 & 2.778 & $0.096^{\#}$ \\
\hline & & $\%$ & $12.5 \%$ & $0.0 \%$ & $10.0 \%$ & & \\
\hline Trunk & Present & Count & 14 & 1 & 15 & 1.961 & $0.292^{\#}$ \\
\hline & & $\%$ & $17.5 \%$ & $5.0 \%$ & $15.0 \%$ & & \\
\hline Upper limb & Present & Count & 20 & 2 & 22 & 2.098 & $0.228^{\#}$ \\
\hline & & $\%$ & $25.0 \%$ & $10.0 \%$ & $22.0 \%$ & & \\
\hline Lower limb & Present & Count & 31 & 4 & 35 & 2.473 & $0.189^{\#}$ \\
\hline & & $\%$ & $38.8 \%$ & $20.0 \%$ & $35.0 \%$ & & \\
\hline Multiple sites & Present & Count & 4 & 13 & 17 & 40.822 & $0.0005^{* *}$ \\
\hline & & $\%$ & $5.0 \%$ & $65.0 \%$ & $17.0 \%$ & & \\
\hline & Com & $n$ of vital & mperatur & mortality & aired $t$-tes & & \\
\hline Mortality & & $N$ & Mean & $S D$ & t-value & & \\
\hline On admission & Dead & 20 & 100.75 & 1.16 & 6.346 & $0.0005^{* *}$ & \\
\hline & Alive & 80 & 99.14 & 0.98 & & & \\
\hline
\end{tabular}

Contd... 
Contd...

\begin{tabular}{|c|c|c|c|c|c|c|c|}
\hline & & & \multicolumn{2}{|c|}{ Mortality } & \multirow[b]{2}{*}{ Total } & \multirow[b]{2}{*}{$x^{2}$-value } & \multirow[b]{2}{*}{ pvalue } \\
\hline & & & Alive & Dead & & & \\
\hline \multirow[t]{2}{*}{ After $48-72$ hours } & Dead & 20 & 100.05 & 0.60 & 13.297 & $0.0005^{* *}$ & \\
\hline & Alive & 80 & 97.86 & 0.67 & & & \\
\hline \multicolumn{8}{|c|}{ Comparison of vital sign-pulse with mortality by unpaired $t$-test } \\
\hline Mortality & & $N$ & Mean & $S D$ & $t$-value & \multicolumn{2}{|c|}{$p$ value } \\
\hline \multirow[t]{2}{*}{ On admission } & Dead & 20 & 95.45 & 2.78 & 0.370 & \multirow[t]{2}{*}{$0.713^{\#}$} & \\
\hline & Alive & 80 & 95.20 & 2.69 & & & \\
\hline \multirow[t]{2}{*}{ After $48-72$ hours } & Dead & 20 & 101.95 & 6.92 & 11.042 & $0.0005^{* *}$ & \\
\hline & Alive & 80 & 84.44 & 3.07 & & & \\
\hline \multicolumn{8}{|c|}{ Comparison between lab findings - WBC with mortality } \\
\hline \multirow[t]{4}{*}{ On admission } & $<25,000$ & Count & 64 & 0 & 64 & 44.444 & $0.0005^{* *}$ \\
\hline & & $\%$ & $80.0 \%$ & $0.0 \%$ & $64.0 \%$ & & \\
\hline & $>25,000$ & Count & 16 & 20 & 36 & & \\
\hline & & $\%$ & $20.0 \%$ & $100.0 \%$ & $36.0 \%$ & & \\
\hline \multirow[t]{4}{*}{ After $48-72$ hours } & $<25,000$ & Count & 80 & 0 & 80 & 100.000 & $0.0005^{* *}$ \\
\hline & & $\%$ & $100.0 \%$ & $0.0 \%$ & $80.0 \%$ & & \\
\hline & $>25,000$ & Count & 0 & 20 & 20 & & \\
\hline & & $\%$ & $0.0 \%$ & $100.0 \%$ & $20.0 \%$ & & \\
\hline \multicolumn{8}{|c|}{ Comparison between lab findings_-creatinine with mortality } \\
\hline \multirow[t]{4}{*}{ On admission } & $<1.6$ & Count & 61 & 0 & 61 & 39.103 & $0.0005^{* *}$ \\
\hline & & $\%$ & $76.3 \%$ & $0.0 \%$ & $61.0 \%$ & & \\
\hline & $>1.6$ & Count & 19 & 20 & 39 & & \\
\hline & & $\%$ & $23.8 \%$ & $100.0 \%$ & $39.0 \%$ & & \\
\hline \multirow[t]{4}{*}{ After $48-72$ hours } & $<1.6$ & Count & 76 & 0 & 76 & 79.167 & $0.0005^{* *}$ \\
\hline & & $\%$ & $95.0 \%$ & $0.0 \%$ & $76.0 \%$ & & \\
\hline & $>1.6$ & Count & 4 & 20 & 24 & & \\
\hline & & $\%$ & $5.0 \%$ & $100.0 \%$ & $24.0 \%$ & & \\
\hline \multicolumn{8}{|c|}{ Comparison between treatment with mortality } \\
\hline \multirow[t]{2}{*}{ Incision and drainage } & & Count & 40 & 0 & 40 & 16.667 & $0.0005^{* *}$ \\
\hline & & $\%$ & $50.0 \%$ & $0.0 \%$ & $40.0 \%$ & & \\
\hline \multirow[t]{2}{*}{ Debridement } & & Count & 23 & 7 & 30 & 0.298 & $0.585^{\#}$ \\
\hline & & $\%$ & $28.8 \%$ & $35.0 \%$ & $30.0 \%$ & & \\
\hline \multirow[t]{2}{*}{ Fasciotomy } & & Count & 13 & 7 & 20 & 3.516 & $0.061^{\#}$ \\
\hline & & $\%$ & $16.3 \%$ & $35.0 \%$ & $20.0 \%$ & & \\
\hline \multirow[t]{2}{*}{ Amputation } & & Count & 4 & 6 & 10 & 11.111 & $0.004^{* *}$ \\
\hline & & $\%$ & $5.0 \%$ & $30.0 \%$ & $10.0 \%$ & & \\
\hline \multirow[t]{2}{*}{ Severe sepsis } & & Count & 0 & 20 & 20 & 100.000 & $0.0005^{* *}$ \\
\hline & & $\%$ & $0.0 \%$ & $100.0 \%$ & $20.0 \%$ & & \\
\hline \multicolumn{8}{|c|}{ Comparison of length of hospital stay with mortality by unpaired $t$-test } \\
\hline Mortality & & $N$ & Mean & $S D$ & $t$-value & & \\
\hline \multirow[t]{2}{*}{ Length of hospital stay } & Dead & 20 & 22 & 3.83 & 13.115 & $0.0005^{* *}$ & \\
\hline & Alive & 80 & 8 & 4.92 & & & \\
\hline
\end{tabular}

\#No statistical significance at $p>0.05$

${ }^{* *}$ Highly significant at $p<0.01$

All clinically and statistically significant variables were included in the multivariate analysis model which demonstrated that the predictors of mortality among patients of NF were patients of age $>60$ years [risk ratio $(\mathrm{RR})=58.57,95 \% \mathrm{Cl}=3.64-941.85, p=$ 0.004], having $\mathrm{DM}(\mathrm{RR}=28.70,95 \% \mathrm{Cl}=1.78-461.51, p=0.017)$, gangrene $(\mathrm{RR}=3.86,95 \% \mathrm{Cl}=1.91-7.75, p=0.0002)$, severe sepsis $(\mathrm{RR}=158.14,95 \% \mathrm{Cl}=9.96-2508.89, p=0.0003)$, involvement of multiple sites ( $\mathrm{RR}=9.06,95 \% \mathrm{Cl}=4.25-19.31, p=0.0001)$, total leukocyte count $(\mathrm{RR}=158.14,95 \% \mathrm{Cl}=9.96-2508.89, p=0.0003)$, and serum creatinine $(R R=126.28,95 \% \mathrm{Cl}=7.92-2013.12$, $p=0.0006$ ) on admission and within 48 to 72 hours of admission.

\section{Comparison of Factors between Amputation and Non-amputation Group}

Patient demographic and clinical characteristics including underlying morbidity like gout, wound appearance such as 
Table 2: Independent predictors of mortality

\begin{tabular}{llll}
\hline \multicolumn{4}{c}{ Risk ratio of mortality } \\
\hline Risk factors & $R R$ & $95 \%$ Cl & p value \\
\hline Swelling & 2.56 & $0.17-37.42$ & $0.491^{\#}$ \\
Skin necrosis & 1.26 & $0.55-2.83$ & $0.582^{\#}$ \\
Gangrene & 3.86 & $1.91-7.75$ & $0.0002^{* *}$ \\
Diabetes mellitus & 28.70 & $1.78-461.51$ & $0.017^{*}$ \\
WBC-after 48-72 hours & 158.14 & $9.96-2508.89$ & $0.0003^{* *}$ \\
Creatinine-after 48-72 hours & 126.28 & $7.92-2013.12$ & $0.0006^{* *}$ \\
Age $>$ 60 years & 58.57 & $3.64-941.85$ & $0.004^{* *}$ \\
Severe sepsis & 158.14 & $9.96-2508.89$ & $0.0003^{* *}$ \\
Multiple sites & 9.06 & $4.25-19.31$ & $0.0001^{* *}$ \\
Lower limb & 0.46 & $0.16-1.28$ & $0.1387^{\#}$ \\
\hline \#o statistical significance at $p>0.05$ & & \\
${ }^{* *}$ Highly significant at $p<0.01$ & & & \\
${ }^{*}$ Statistical significant at $p<0.05$ & & &
\end{tabular}

Table 3: Comparison of factors between amputation and non-amputation group

\begin{tabular}{|c|c|c|c|c|c|c|c|}
\hline & & & \multicolumn{2}{|c|}{ Outcome } & \multirow[b]{2}{*}{ Total } & \multirow[b]{2}{*}{$x^{2}$-value } & \multirow[b]{2}{*}{$p$ value } \\
\hline & & & Non-amputation & Amputation & & & \\
\hline \multicolumn{8}{|c|}{ Comparison between age with amputation } \\
\hline \multirow[t]{4}{*}{ Age } & $<60$ & Count & 55 & 4 & 59 & 35.976 & $0.310^{\#}$ \\
\hline & & $\%$ & $61.1 \%$ & $40.0 \%$ & $59.0 \%$ & & \\
\hline & $>60$ & Count & 35 & 6 & 41 & & \\
\hline & & $\%$ & $38.9 \%$ & $60.0 \%$ & $41.0 \%$ & & \\
\hline \multicolumn{8}{|c|}{ Comparison between gender with amputation } \\
\hline \multirow[t]{4}{*}{ Gender } & Female & Count & 29 & 6 & 35 & 3.053 & $0.094^{\#}$ \\
\hline & & $\%$ & $32.2 \%$ & $60.0 \%$ & $35.0 \%$ & & \\
\hline & Male & Count & 61 & 4 & 65 & & \\
\hline & & $\%$ & $67.8 \%$ & $40.0 \%$ & $65.0 \%$ & & \\
\hline \multicolumn{8}{|c|}{ Comparison between underlying morbidities with amputation } \\
\hline \multirow{2}{*}{\multicolumn{2}{|c|}{ Diabetes mellitus }} & Count & 52 & 7 & 59 & 0.556 & $0.520^{\#}$ \\
\hline & & $\%$ & $57.8 \%$ & $70.0 \%$ & $59.0 \%$ & & \\
\hline \multirow{2}{*}{\multicolumn{2}{|c|}{ Coronary heart disease }} & Count & 9 & 1 & 10 & 0.000 & $1.000^{\#}$ \\
\hline & & $\%$ & $10.0 \%$ & $10.0 \%$ & $10.0 \%$ & & \\
\hline \multirow{2}{*}{\multicolumn{2}{|c|}{ Renal disease }} & Count & 6 & 0 & 6 & 0.709 & $1.000^{\#}$ \\
\hline & & $\%$ & $6.7 \%$ & $0.0 \%$ & $6.0 \%$ & & \\
\hline \multirow[t]{2}{*}{ Cirrhosis } & & Count & 4 & 1 & 5 & 0.585 & $0.416^{\#}$ \\
\hline & & $\%$ & $4.4 \%$ & $10.0 \%$ & $5.0 \%$ & & \\
\hline \multirow[t]{2}{*}{ Gout } & & Count & 11 & 4 & 15 & 5.447 & $0.041^{*}$ \\
\hline & & $\%$ & $12.2 \%$ & $40.0 \%$ & $15.0 \%$ & & \\
\hline \multicolumn{8}{|c|}{ Comparison between wound appearance with amputation } \\
\hline \multirow[t]{2}{*}{ Swelling } & Count & 85 & 10 & 95 & 0.585 & $1.000^{\#}$ & \\
\hline & $\%$ & $94.4 \%$ & $100.0 \%$ & $95.0 \%$ & & & \\
\hline \multirow[t]{2}{*}{ Redness } & Count & 90 & 0 & 90 & 100.000 & $0.0005^{* *}$ & \\
\hline & $\%$ & $100.0 \%$ & $0.0 \%$ & $90.0 \%$ & & & \\
\hline \multirow[t]{2}{*}{ Bleb } & Count & 40 & 0 & 40 & 7.407 & $0.005^{* *}$ & \\
\hline & $\%$ & $44.4 \%$ & $0.0 \%$ & $40.0 \%$ & & & \\
\hline \multirow[t]{2}{*}{ Skin necrosis } & Count & 30 & 0 & 30 & 4.762 & $0.030^{*}$ & \\
\hline & $\%$ & $33.3 \%$ & $0.0 \%$ & $30.0 \%$ & & & \\
\hline \multirow[t]{2}{*}{ Gangrene } & Count & 0 & 10 & 10 & 100.000 & $0.0005^{* *}$ & \\
\hline & $\%$ & $0.0 \%$ & $100.0 \%$ & $10.0 \%$ & & & \\
\hline
\end{tabular}


Contd...

\begin{tabular}{|c|c|c|c|c|c|c|c|}
\hline & & & \multicolumn{2}{|c|}{ Outcome } & \multirow[b]{2}{*}{ Total } & \multirow[b]{2}{*}{$x^{2}$-value } & \multirow[b]{2}{*}{$p$ value } \\
\hline & & & Non-amputation & Amputation & & & \\
\hline \multirow[t]{2}{*}{ Severe pain } & Count & 65 & 10 & 75 & 3.704 & $0.062^{\#}$ & \\
\hline & $\%$ & $72.2 \%$ & $100.0 \%$ & $75.0 \%$ & & & \\
\hline \multicolumn{8}{|c|}{ Comparison between the site of wound with amputation } \\
\hline \multirow[t]{2}{*}{ Head and neck } & & Count & 10 & 0 & 10 & 1.235 & $0.592^{\#}$ \\
\hline & & $\%$ & $11.1 \%$ & $0.0 \%$ & $10.0 \%$ & & \\
\hline \multirow[t]{2}{*}{ Trunk } & & Count & 15 & 0 & 15 & 1.961 & $0.351^{\#}$ \\
\hline & & $\%$ & $16.7 \%$ & $0.0 \%$ & $15.0 \%$ & & \\
\hline \multirow[t]{2}{*}{ Upper limb } & & Count & 18 & 4 & 22 & 2.098 & $0.220^{\#}$ \\
\hline & & $\%$ & $20.0 \%$ & $40.0 \%$ & $22.0 \%$ & & \\
\hline \multirow[t]{2}{*}{ Lower limb } & & Count & 29 & 6 & 35 & 3.053 & $0.094^{\#}$ \\
\hline & & $\%$ & $32.2 \%$ & $60.0 \%$ & $35.0 \%$ & & \\
\hline \multirow[t]{2}{*}{ Multiple sites } & & Count & 17 & 0 & 17 & 2.276 & $0.204^{\#}$ \\
\hline & & $\%$ & $18.9 \%$ & $0.0 \%$ & $17.0 \%$ & & \\
\hline \multicolumn{8}{|c|}{ Comparison of vital sign-temperature $(\mathrm{F})$ with amputation by unpaired $t$-test } \\
\hline Outcome & & $N$ & Mean & $S D$ & $t$-value & & pvalue \\
\hline \multirow[t]{2}{*}{ On admission } & Amputation & 10 & 100.20 & 1.62 & 2.088 & $0.039^{*}$ & \\
\hline & Non-amputation & 90 & 99.38 & 1.13 & & & \\
\hline \multirow[t]{2}{*}{ After $48-72$ hours } & Amputation & 10 & 99.40 & 1.07 & 3.533 & $0.001^{* *}$ & \\
\hline & Non-amputation & 90 & 98.18 & 1.03 & & & \\
\hline \multicolumn{8}{|c|}{ Comparison of vital signs pulse with amputation by unpaired $t$-test } \\
\hline Outcome & & $N$ & Mean & $S D$ & $t$-value & & pvalue \\
\hline \multirow[t]{2}{*}{ On admission } & Amputation & 10 & 95.20 & 2.90 & 0.062 & $0.951^{\#}$ & \\
\hline & Non-amputation & 90 & 95.26 & 2.69 & & & \\
\hline \multirow[t]{2}{*}{ After $48-72$ hours } & Amputation & 10 & 93.50 & 9.59 & 2.326 & $0.022^{*}$ & \\
\hline & Non-amputation & 90 & 87.32 & 7.78 & & & \\
\hline \multicolumn{8}{|c|}{ Comparison between lab findings-WBC with amputation } \\
\hline \multirow[t]{4}{*}{ On admission } & $<25,000$ & Count & 64 & 0 & 64 & 19.753 & $0.0005^{* *}$ \\
\hline & & $\%$ & $71.1 \%$ & $0.0 \%$ & $64.0 \%$ & & \\
\hline & $>25,000$ & Count & 26 & 10 & 36 & & \\
\hline & & $\%$ & $28.9 \%$ & $100.0 \%$ & $36.0 \%$ & & \\
\hline \multirow[t]{4}{*}{ After $48-72$ hours } & $<25,000$ & Count & 76 & 4 & 80 & 11.111 & $0.004^{* *}$ \\
\hline & & $\%$ & $84.4 \%$ & $40.0 \%$ & $80.0 \%$ & & \\
\hline & $>25,000$ & Count & 14 & 6 & 20 & & \\
\hline & & $\%$ & $15.6 \%$ & $60.0 \%$ & $20.0 \%$ & & \\
\hline & & parison $\mathrm{k}$ & en lab findings $-c$ & atinine with ar & tation & & \\
\hline On admission & $<1.6$ & Count & 61 & 0 & 61 & 17.379 & $0.0005^{* *}$ \\
\hline & & $\%$ & $67.8 \%$ & $0.0 \%$ & $61.0 \%$ & & \\
\hline & $>1.6$ & Count & 29 & 10 & 39 & & \\
\hline & & $\%$ & $32.2 \%$ & $100.0 \%$ & $39.0 \%$ & & \\
\hline After $48-72$ hours & $<1.6$ & Count & 76 & 0 & 76 & 35.185 & $0.0005^{* *}$ \\
\hline & & $\%$ & $84.4 \%$ & $0.0 \%$ & $76.0 \%$ & & \\
\hline & $>1.6$ & Count & 14 & 10 & 24 & & \\
\hline & & Comp & between treatmer & with amputat & & & \\
\hline Incision and drainage & & Count & 40 & 0 & 40 & 7.407 & $0.005^{* *}$ \\
\hline & & $\%$ & $44.4 \%$ & $0.0 \%$ & $40.0 \%$ & & \\
\hline Debridement & & Count & 30 & 0 & 30 & 4.762 & $0.030^{*}$ \\
\hline & & $\%$ & $33.3 \%$ & $0.0 \%$ & $30.0 \%$ & & \\
\hline Fasciotomy & & Count & 20 & 0 & 20 & 2.778 & $0.205^{\#}$ \\
\hline & & $\%$ & $22.2 \%$ & $0.0 \%$ & $20.0 \%$ & & \\
\hline
\end{tabular}


Contd...

\begin{tabular}{|c|c|c|c|c|c|c|c|}
\hline & & & \multicolumn{2}{|c|}{ Outcome } & \multirow[b]{2}{*}{ Total } & \multirow[b]{2}{*}{$x^{2}$-value } & \multirow[b]{2}{*}{$p$ value } \\
\hline & & & Non-amputation & Amputation & & & \\
\hline \multirow{2}{*}{\multicolumn{2}{|c|}{ Amputation }} & Count & 0 & 10 & 10 & 100.000 & $0.0005^{* *}$ \\
\hline & & $\%$ & $0.0 \%$ & $100.0 \%$ & $10.0 \%$ & & \\
\hline \multirow[t]{2}{*}{ Severe sepsis } & & Count & 14 & 6 & 20 & 11.111 & $0.004^{* *}$ \\
\hline & & $\%$ & $15.6 \%$ & $60.0 \%$ & $20.0 \%$ & & \\
\hline \multicolumn{8}{|c|}{ Comparison of length of hospital stay with amputation by unpaired $t$-test } \\
\hline Outcome & & $N$ & Mean & $S D$ & $t$-value & & $p$ value \\
\hline \multirow{2}{*}{$\begin{array}{l}\text { Length of hospital } \\
\text { stay }\end{array}$} & Amputation & 10 & 18 & 8.66 & 3.527 & $0.001^{* *}$ & \\
\hline & Non-amputation & 89 & 10 & 6.55 & & & \\
\hline
\end{tabular}

\#No statistical significance at $p>0.05$

${ }^{* *}$ Highly significant at $p<0.01$

*Statistical significant at $p<0.05$

Table 4: Independent predictors of amputation

\begin{tabular}{llll}
\hline \multicolumn{4}{c}{ Risk ratio of amputation } \\
\hline Risk factors & $R R$ & $95 \% \mathrm{Cl}$ & p value \\
\hline Swelling & 1.31 & $0.08-19.81$ & $0.844^{\#}$ \\
Skin necrosis & 0.11 & $0.00-1.80$ & $0.121^{\#}$ \\
Gangrene & 173.73 & $10.91-2764.80$ & $0.0003^{* *}$ \\
Diabetes mellitus & 1.62 & $0.44-5.90$ & $0.463^{\#}$ \\
WBC-after 48-72 hours & 6.00 & $1.86-19.26$ & $0.002^{* *}$ \\
Creatinine-after 48-72 hours & 64.68 & $3.92-1064.68$ & $0.003^{* *}$ \\
Age >60 years & 2.15 & $0.64-7.17$ & $0.209^{\#}$ \\
Severe sepsis & 6.00 & $1.86-19.26$ & $0.002^{* *}$ \\
Multiple sites & 0.22 & $0.01-3.62$ & $0.290^{\#}$ \\
Lower limb & 2.78 & $0.84-9.21$ & $0.093^{\#}$ \\
\hline
\end{tabular}

\#No statistical significance at $p>0.05$

**Highly significant at $p<0.01$

redness, blebs, gangrene, skin necrosis, WBC count $(>25,000)$, serum creatinine level $(>1.6)$ on admission and within 48-72 hours after admission, severe sepsis and length of hospital stay showed statistically significant differences ( $p$ value $<0.05$ ) between amputation and non-amputation groups. Other factors did not differ significantly between the two groups.

All clinically and statistically significant variables were included in the multivariate analysis model which demonstrated that the independent predictors of amputation among patients of NF included having gangrene $(\mathrm{RR}=173.73,95 \% \mathrm{Cl}=10.91-2764.80, p$ $=0.0003)$, severe sepsis $(\mathrm{RR}=6.00,95 \% \mathrm{Cl}=1.86-19.26, p=0.002)$, total leukocyte count $(\mathrm{RR}=6.00,95 \% \mathrm{Cl}=1.86-19.26, p=0.002)$, and serum creatinine $(\mathrm{RR}=64.68,95 \% \mathrm{Cl}=3.92-1064.68, p=0.003)$ on admission and within 48 to 72 hours of admission.

\section{Discussion}

Necrotizing fasciitis was initially described as gangrene of the subcutaneous tissue which was spreading very, rapidly caused by beta-hemolytic streptococci group I and later being considered as a clinical entity rather than a specific bacterial infection. Necrotizing fasciitis is a surgical emergency. Early recognition and prompt aggressive surgical debridement of all necrotic tissue are critical for survival. $^{9}$
Diagnosing NF early is essential in stopping the progression of this disease and for achieving a better prognosis. Late detection almost leads to a grave prognosis. We diagnose NF, whenever necrosis of subcutaneous tissues occurs extending through the fascial planes. Scarce cutaneous findings early in the course of the disease make it even more difficult to diagnose this condition. Often the disease is masqueraded as cellulitis or abscesses. In these patients, the diagnosis was made when the infection progressed despite treatment with (broad-spectrum) intravenous antibiotics. Many studies that were conducted in the past concluded that recognizing the disease early with early surgical intervention prevents morbidity and mortality in patients with NF. It is difficult to differentiate between NF from other soft tissue infections such as cellulitis due to minimum specific cutaneous signs, making its diagnosis even more difficult.

Our study involved a prospective assessment of various risk factors that serve as independent predictors of amputation and mortality in patients with NF. Our results should indicate the ways and means for early recognition of NF, to preserve limb from amputation and reduce mortality. Necrotizing fasciitis being an emergency surgery condition having a high mortality rate and with sufficient treatment also, the reported rate of mortality varying from 6 to $36 \% .{ }^{10}$ The overall mortality rate in our study was $20 \%$ of 100 patients and the rate of amputation was $10 \%$. These patients had adverse outcomes, and although the cases were investigated and treated with broad-spectrum antibiotics, these patients underwent amputation or died rapidly from systemic inflammatory response syndrome (SIRS). ${ }^{10}$ However, identification of independent risk factors of amputation and death could affect the survival and preservation of limbs of patients which could assist the surgeons, to counter these risk factors for achieving a successful outcome of treatment in all patients. Our study identified gangrene, severe sepsis, total leukocyte count $>25,000$, and serum creatinine values $>1.6 \mathrm{mg} / \mathrm{dL}$ on admission as well as within 48-72 hours, as independent predictors for both amputation and mortality and some other factors like age $>60$ years, the involvement of multiple sites, DM mainly predicting the mortality rate independently. Previous studies found that being female, having DM, cutaneous gangrene noted on admission, evidence of an underlying condition, heart disease, serum creatinine $>1.6$, and shock (systolic blood pressure, $90 \mathrm{~mm} \mathrm{Hg}$ ) at hospital admission were independent predictors for limb loss 
and mortality in patients with NF. ${ }^{11}$ In our study, the association of DM related to mortality is consistent with that of a previous study reported by Dworkin et al. ${ }^{11}$ Some independent studies concluded that although DM was the most frequently occurring comorbidity in NF patients, significantly it did not increase amputation or mortality rates. ${ }^{12}$ Diabetes mellitus was also the most common comorbidity in our patients, as reported by other investigators. ${ }^{13}$ The pathophysiology of DM is related to the worst outcomes of NF because hyperglycemia induces bacterial growth and tissue ischemia as a result of peripheral vascular disease, leading to amputation and mortality. ${ }^{14}$ Patients with DM often develop severe atherosclerosis. The atherosclerotic changes occurring in the limb vessels were found to be more extensive in small-caliber arteries, resulting in ischemia and gangrene, ultimately leading to amputation or mortality. ${ }^{15}$ Nearly $45 \%$ of sites of amputation which were involving fingers or toes, distinguishing between wet gangrene of the DM foot from NF, which sometimes occur simultaneously could not be differentiated. Elderly persons with or without underlying diseases, such as chronic heart disease and liver cirrhosis, with the involvement of multiple sites in disease, were considered as having worse prognostic factors, similar to previous studies reporting that advanced age was associated with an increased risk of mortality. ${ }^{11,12}$ This study found that age being $>60$ years, increased the mortality rate significantly. However, some studies found that advanced age did not affect mortality. ${ }^{10}$

Necrotizing fasciitis presenting with severe sepsis, with total leukocyte count $>25,000$ on admission as well as within $48-72$ hours of admission and gangrene at the time of diagnosis was found to be significantly predictive for mortality $(p=0.0003, p=0.0003, p$ $=0.0002$, respectively) and amputation ( $p=0.002, p=0.002, p=$ 0.0003 , respectively) in our study. Dworkin et al. ${ }^{11}$ also reported that cutaneous gangrene which was present on admission was significantly ( $p=0.005)$ associated with increased risk of loss of limb. In previous studies, the presence of gangrene was observed in latestage NF and was the leading cause of an adverse outcome. ${ }^{16}$ As in other previous studies, ${ }^{17}$ we found that soft tissue swelling of the affected area was a common clinical manifestation in most patients with NF. The infection which spreads into lymphatic and vascular systems causes tissue edema and muscle necrosis, and eventually, skin necrosis will occur consequent to thrombosis of microvascular vessels and dysfunction of the nerve supply. ${ }^{18}$ Gangrene is tissue necrosis due to lack of blood supply. Whenever gangrene was presented, debridement or amputation was needed depending on the extension of necrotic areas. ${ }^{15}$

Our study found that serum creatinine values $>1.6 \mathrm{mg} / \mathrm{dL}$ posed an increased risk for limb loss and death. An increase in the serum creatinine levels reflects renal dysfunction, which may be associated with septic shock. Anaya et al. ${ }^{19}$ reported that shock (systolic blood pressure, $90 \mathrm{~mm} \mathrm{Hg}$ ) at admission was an independent predictor for limb loss, raised creatinine level, and higher mortality similar to many previous studies. ${ }^{12}$ However, as reported by Dos Santos et al. ${ }^{20}$ serum creatinine levels were not associated with major amputation.

As per literature, prognostic factors to evaluate the severity of NF patient included the LRINEC (laboratory risk indicator for NF) score, composed of six marker variables, including C-reactive protein, total white blood cell count, hemoglobin, serum sodium, serum creatinine, and serum glucose; ${ }^{9}$ and acute physiology and chronic health evaluation II (APACHE II) scores were including the patient's vital signs (temperature, mean arterial pressure, heart rate, and respiratory rate), oxygenation $\left[\mathrm{A}-\mathrm{PaO}_{2}\left(\mathrm{FiO}_{2}\right.\right.$ 0.50\%) or $\mathrm{PaO}_{2}$ $\left(\mathrm{FiO}_{2}, 50 \%\right)$ ], metabolic parameters [sodium, potassium, creatinine, bicarbonate concentrations (arterial $\mathrm{pH}$ or $\mathrm{HCO}_{3}$ ), hematocrit, white blood cell count] were used as a prognostic scoring system for critical care, and Glasgow coma score and calculates a score relating to the severity of the disease of a patient who was admitted to the ICU. ${ }^{21}$

The limitations of our study include that neither of these scoring systems could be applied (LRINEC and APACHE II). Despite the limitations of our study, the results of our study might be useful in clinical practice while caring for NF patients in provincial hospitals and physicians can make use of the risk factors for evaluating the patients despite limited resources that are available at local hospitals.

\section{Conclusion}

In our study, we concluded that gangrene, severe sepsis, total leukocyte count $>25,000$, and serum creatinine values $>1.6 \mathrm{mg} /$ $\mathrm{dL}$ on admission as well as within 48-72 hours, as independent predictors for both amputation and mortality and some other factors like age $>60$ years, the involvement of multiple sites, DM mainly predicting the mortality rate independently. Thus for patients presenting with any of these clinical predictors, the treating surgeon should have direct concern toward the progression of the disease and might be considered for early investigation and close monitoring to prevent morbidity and mortality as well as limb loss as much as possible.

\section{References}

1. Kihiczak GG, Schwartz RA, Kapila R. Necrotizing fasciitis: a deadly infection. J Eur Acad Dermatol Venereol 2006;20(4):365-369. DOI: 10.1111/j.1468-3083.2006.01487.x.

2. Salcido RS. Necrotizing fasciitis: reviewing the causes and treatment strategies. Adv Skin Wound Care 2007;20(5):288-293. DOI: 10.1097/01. ASW.0000269317.76380.3b.

3. Tang $\mathrm{S}, \mathrm{K}$ wok TK, Ho PL, et al. Necrotizing fasciitis in a renal transplant recipient treated with FK 506: the first reported case. Clin Nephrol 2001;56(6):481-485.

4. Tang WM, Ho PL, Fung KK, et al. Necrotising fasciitis of a limb. J Bone Joint Surg Br 2001;83(5):709-714. DOI: 10.1302/0301620X.83B5.0830709.

5. Trent JT, Kirsner RS. Diagnosing necrotizing fasciitis. Adv Skin Wound Care 2002;15(3):135-138. DOI: 10.1097/00129334-20020500000010.

6. Cainzos M, Gonzalez-Rodriguez FJ. Necrotizing soft tissue infections. Curr Opin Crit Care 2007;13(4):433-439. DOI: 10.1097/ MCC.0b013e32825a6a1b.

7. Fontes Jr RA, Ogilvie CM, Miclau T. Necrotizing soft-tissue infections. J Am Acad Orthop Surg 2000;8(3):151-158. DOI: 10.5435/00124635200005000-00002.

8. Perry BN, Floyd III WE. Gas gangrene and necrotizing fasciitis in the upper extremity. J Surg Orthop Adv 2004;13(2):57-68.

9. Su YC, Chen HW, Hong YC, et al. Laboratory risk indicator for necrotizing fasciitis score and the outcomes. ANZ J Surg 2008;78(11):968-972. DOI: 10.1111/j.1445-2197.2008.04713.x.

10. McHenry CR, Piotrowski JJ, Petrinic $D$, et al. Determinants of mortality for necrotizing soft-tissue infections. Ann Surg 1995;221(5):558-563. DOI: 10.1097/00000658-199505000-00013.

11. Dworkin MS, Westercamp MD, Park L, et al. The epidemiology of necrotizing fasciitis including factors associated with death and amputation. Epidemiol Infect 2009;137(11):1609-1614. DOI: 10.1017/ S0950268809002532. 
12. Bair MJ, Chi H, Wang WS, et al. Necrotizing fasciitis in southeast Taiwan: clinical features, microbiology, and prognosis. Int J Infect Dis 2009;13(2):255-260. DOI: 10.1016/j.ijid.2008. 04.015 .

13. Bucca K, Spencer R, Orford N, et al. Early diagnosis and treatment of necrotizing fasciitis can improve survival: an observational intensive care unit cohort study. ANZ J Surg 2013;83(5):365-370. DOI: 10.1111/j.1445-2197.2012.06251.x.

14. Nakayama J, Busse R. An analysis of vulvar necrotizing fasciitis in the unique and ethnically diverse Hawaiian population. Hawaii Med J 2010;69(1):13-16.

15. Falkel JE. Amputation as a consequence of diabetes mellitus. An Epidemiolog Rev Phys Ther 1983;63(6):960-964. DOI: 10.1093/ ptj/63.6.960.

16. Hongladaromp C, Chareonsil B, Phadhana-Anake O. Predictors on mortality from necrotizng fasciitis in Sawanpracharak hospital, Nakhonsawan, Thailand. Chiang Mai Med J 2009;48(4) 135-142.

17. Awsakulsutthi S. A retrospective review of necrotizing fasciitis in Thammasat University Hospital. J Med Assoc Thai 2010;93(Suppl 7):S246-S253.

18. Puvanendran R, Huey JC, Pasupathy S. Necrotizing fasciitis. Can FamPhysician 2009;55:981-987.

19. Anaya DA, McMahon $K$, Nathens $A B$, et al. Predictors of mortality and limb loss in necrotizing soft tissue infections. Arch Surg 2005;140(2):151-157. DOI: 10.1001/archsurg.140.2.151.

20. Dos Santos VP, da Silveira DR, Caffaro RA. Risk factors for primary major amputation in diabetic patients. Sao Paulo Med J 2006;124(2):66-70. DOI: 10.1590/S1516-31802006000200004.

21. Knaus WA, Draper EA, Wagner DP, et al. APACHE II: a severity of disease classification system. Crit Care Med 1985;13(10):818-829. DOI: 10.1097/00003246-198510000-00009. 